\title{
EXACT MONTE CARLO SIMULATION OF KILLED DIFFUSIONS
}

\author{
BRUNO CASELLA* AND \\ GARETH O. ROBERTS, ${ }^{* *}$ University of Warwick
}

\begin{abstract}
We describe and implement a novel methodology for Monte Carlo simulation of onedimensional killed diffusions. The proposed estimators represent an unbiased and efficient alternative to current Monte Carlo estimators based on discretization methods for the cases when the finite-dimensional distributions of the process are unknown. For barrier option pricing in finance, we design a suitable Monte Carlo algorithm both for the single barrier case and the double barrier case. Results from numerical investigations are in excellent agreement with the theoretical predictions.
\end{abstract}

Keywords: Monte Carlo; diffusions; exact algorithm

2000 Mathematics Subject Classification: Primary 65CO5

\section{Introduction}

Many important problems can be reduced to the computation of the expected value, say $v$, of a functional of a diffusion process $X$. In this paper we will derive and implement efficient and unbiased methods for Monte Carlo evaluation of $v$ when analytic expressions for the finitedimensional distributions of $X$ are not available and the value of the functional depends on barriers (either a single barrier or double barriers).

We consider a one-dimensional diffusion process $X$ :

$$
\mathrm{d} X_{t}=\mu\left(X_{t}\right) \mathrm{d} t+\sigma\left(X_{t}\right) \mathrm{d} W_{t}, \quad X_{0}=x_{0}, 0 \leq t \leq T,
$$

where $\left\{W_{t}: 0 \leq t \leq T\right\}$ is a standard Brownian Motion, and the drift $\mu$ and the diffusion coefficient $\sigma$ are presumed to satisfy the usual conditions that guarantee the existence of a weakly unique global solution of (1) (see, e.g. Chapter 5 of Øksendal (1998)).

Let $H:=(a, b)$ be an open interval of $\mathbb{R}$ such that $X_{0}=x_{0} \in H$. We are interested in the computation of

$$
v:=\mathrm{E}\left[h\left(X_{T}\right) \mathbf{1}_{\{\tau>T\}} \mid X_{0}=x_{0}\right],
$$

where $T>0$ is a fixed time, $\tau$ is the first exit time of $X$ from the set $H$, and $h(\cdot)$ is a measurable function.

In mathematical finance the problem of the computation of (2) arises in many contexts, for example, in barrier options pricing (see, e.g. Merton (1973) and Reiner and Rubinstein (1991)) or in structural credit risk modelling (see, e.g. Black and Cox (1976) and Longstaff and Schwarz (1995)). Analytic computation of (2) is only possible for a limited collection of

Received 5 January 2007; revision received 11 December 2007.

* Current address: via novara, 31, Milan, 20147, Italy.

** Postal address: Department of Statistics, University of Warwick, Coventry, CV4 7AL, UK.

Email address: gareth.o.roberts@warwick.ac.uk 
simple models. For example, in the Black-Scholes case Reiner and Rubinstein (1991) derived an explicit formula for the price of single barrier options of European style. Recently, Davydov and Linetsky (2001) extended these results to asset processes driven by diffusions of constant elasticity of variance. However, in general we have to approximate (2) by Monte Carlo methods.

\subsection{Background}

In principle, when using Monte Carlo simulation, many trajectories of $X$ are generated and the value of the functional is evaluated at each sample path. Averaging over all paths then provides an unbiased estimator of $v$ which converges to the true value as the number of iterations increases. When the transition densities of $X$ are not known, common practice introduces some kind of discrete approximation $\tilde{X}$ of the process $X$. From the discrete approximation, suitable estimates for expected functions can be derived. The simplest and most popular of these methods is the Euler discretization, which approximates (1) by means of

$$
\tilde{X}_{i \Delta}=\tilde{X}_{(i-1) \Delta}+\mu\left(\tilde{X}_{(i-1) \Delta}\right) \Delta+\sigma\left(\tilde{X}_{(i-1) \Delta}\right) \sqrt{\Delta} \varepsilon_{i}, \quad \tilde{X}_{0}=x_{0}, i=1,2, \ldots, n,
$$

where $n=T / \Delta$ and $\left\{\varepsilon_{i}\right\}_{i=1,2, \ldots, n}$ are independent and identically distributed (i.i.d.) standard normal random variables. Discretization methods introduce bias in the simulation which tends to 0 as $n$ tends to $\infty$ (for fixed $T$ ). Using discretization schemes which are sufficiently fine to ensure bias reduction to acceptable levels may be computationally very expensive. A related problem concerns the optimal allocation of the total computational budget between the number of time steps and the number of simulation trials. Moreover, when dealing with functionals involving barriers, the discretization scheme is subject to two sources of error: one error arising from the actual approximation of (1) by its discretized counterpart, and the other error arising from the use of a discrete exit time instead of a continuous exit time. In fact, Gobet (2000) proved, under rather general conditions, that in the case of killed diffusions the Euler scheme converged weakly at a rate of $1 / \sqrt{n}$ in contrast to $1 / n$ which is customary for sufficiently smooth payoff functions. A widely used technique consists of interpolating the discretized process (3) into a continuous Euler scheme by means of Brownian bridges; i.e. for any $i=1,2, \ldots, n$ and for any $t \in\left[t_{(i-1) \Delta}, t_{i \Delta}\right)$, set

$$
\tilde{X}_{t}=\tilde{X}_{(i-1) \Delta}+\mu\left(\tilde{X}_{(i-1) \Delta}\right)(t-(i-1) \Delta)+\sigma\left(\tilde{X}_{(i-1) \Delta}\right)\left(W_{t}-W_{(i-1) \Delta}\right) .
$$

The idea here is to produce a realisation of the process (3) generating $\tilde{X}_{\Delta}, \tilde{X}_{2 \Delta}, \ldots, \tilde{X}_{n \Delta}$. Then, for any two points $\tilde{X}_{(i-1) \Delta}$ and $\tilde{X}_{i \Delta}$, we sample a [0, 1]-uniformly distributed random variable and compare it with the crossing probability of the corresponding Brownian bridge. Schemes based on Brownian bridge interpolation of the Euler trajectories can be succesfully applied to many simulation problems. Asmussen and Glynn (2007, Section X.8) applied analogous ideas to the exact simulation of reflected Brownian motion with drift and to the approximation of reflected diffusions. These methods improve the rate of convergence of the discrete Euler scheme; in particular, Gobet (2000), (2001) showed that the weak error was of order $n^{-1}$. However, when $n$ is large, the use of (4) for Monte Carlo simulation of $v$ can be computationally expensive, involving the simulation of a very large number of uniform random variables at each iteration of the Monte Carlo algorithm.

\subsection{A new approach}

We will describe and implement a new method for Monte Carlo estimation of $v$. Our method is designed to deal with those cases when the family of transition densities of the process $X$ is not available. In fact, it improves the performances of Monte Carlo algorithms based on discretization methods in two directions. 
- The Monte Carlo estimator is simulated exactly, so no bias is introduced in the simulation. Because of this, we call our method the exact Monte Carlo method.

- There is no trade-off between accuracy of the estimation and computational effort. The higher the level of approximation required, the larger the computational efficiency advantage gained by use of the exact algorithm.

We will see that the exact algorithm approach is actually highly computationally efficient, often requiring less computing effort than rather crude discretization methods.

Our approach builds on recent advances in exact simulation of diffusions. The main idea was introduced in Beskos et al. (2006a). Subsequently, it has been further developed in Beskos et al. (2008) and applied to inference for discretely observed diffusion processes (Beskos et al. (2006b)) and particle filter estimation for diffusions (Fearnhead et al. (2008)). Currently, there are three versions of the exact algorithm: EA1, EA2, and EA3. For simplicity, in the present paper we will focus on the basic one (EA1), although generalisations to EA2 and EA3 are possible. In particular, the generalisation to EA2 will be presented in Section 4. The paper is organised as follows. In Subsection 2.1 we define the class of diffusions of interest. In Section 2, after some preliminaries, we state the mathematical results that justify the Monte Carlo procedure. In Section 3 we describe in details the exact Monte Carlo algorithm. In Section 4 we introduce some further probabilistic constructions supporting more general versions of our algorithm. In Section 5 we report the results of a numerical study comparing the performance of our Monte Carlo estimator with the Monte Carlo estimators generated by (3) and (4). We end in Section 6 with some comments and concluding remarks.

\section{Theoretical framework}

\subsection{The model}

In this subsection we describe the class $\mathscr{D}$ of one-dimensional diffusion processes (1) for which the exact Monte Carlo method can be applied. We introduce the transformed process $Y:=\left\{Y_{t} ; 0 \leq t \leq T\right\}$ defined by

$$
Y_{t}=\eta\left(X_{t}\right)=\int_{z}^{X_{t}} \frac{1}{\sigma(u)} \mathrm{d} u,
$$

where $z$ can be any element of the state space of $X$. Assuming that $\sigma$ is nowhere 0 and continuously differentiable, by Itô's formula, the process $Y$ satisfies the stochastic differential equation (SDE)

$$
\mathrm{d} Y_{t}=\alpha\left(Y_{t}\right) \mathrm{d} t+\mathrm{d} W_{t}, \quad Y_{0}=\eta\left(x_{0}\right)=y_{0},
$$

where

$$
\alpha(u)=\frac{\mu\left(\eta^{-1}(u)\right)}{\sigma\left(\eta^{-1}(u)\right)}-\frac{1}{2} \sigma^{\prime}\left(\eta^{-1}(u)\right)
$$

and $\eta^{-1}$ denotes the inverse transformation. Let $C=C([0, T], \mathbb{R})$ be the set of continuous functions from $[0, T]$ to $\mathbb{R}$, let $\mathcal{C}$ be the $\sigma$-algebra generated by the cylinder subsets of $C$, and let $\left\{\mathcal{C}_{t}: t \in[0, T]\right\}$ be the corresponding filtration. We denote by $\omega=\left\{\omega_{s}: 0 \leq s \leq T\right\}$ the generic element of $C$. Let $\mathrm{Q}$ denote the probability measure induced by the process $Y$ in (6) 
on $(C, \mathcal{C})$ and let $\mathrm{W}$ denote the corresponding measure induced by the Brownian motion with starting point $y_{0}$. We introduce the following conditions on $Y$.

(B0) $\mathrm{Q}<<\mathrm{W}$ and the Girsanov representation holds:

$$
\frac{\mathrm{dQ}}{\mathrm{dW}}(\omega)=\exp \left(\int_{0}^{T} \alpha\left(\omega_{t}\right) \mathrm{d} \omega_{t}-\frac{1}{2} \int_{0}^{T} \alpha^{2}\left(\omega_{t}\right) \mathrm{d} t\right) .
$$

(B1) The drift function $\alpha$ is $\mathcal{C}^{1}$ (differentiable with continuity).

(B2) The function $\left(\alpha^{2}+\alpha^{\prime}\right) / 2$ is bounded.

Conditions (B0)-(B2) define the class $\mathscr{D}$ of diffusions (1) of interest:

$$
\mathscr{D}:=\{X: \eta(X) \text { satisfies conditions (B0)-(B2) }\}
$$

\subsection{Preliminaries}

Roughly speaking, EA1 (Beskos et al. (2006a)) exploits a transformation of the likelihood ratio (7) to allow a rejection sampling on the path space $C$ in order to simulate from the (unknown) diffusion measure Q. Conditions (B1) and (B2) permit the Girsanov ratio to be bounded by an explicit and everywhere finite function of $\omega_{T}$, which in turn permits an appropriate rejection sampling algorithm to be constructed. This construction is described in detail in Beskos and Roberts (2005), Beskos et al. (2006a), and Beskos et al. (2006b). Explicit links between (B1) and (B2) and Conditions 1-3 of Beskos and Roberts (2005, pp. 2425-2426) are provided in Appendix B. EA1 returns as output a partial exact representation of the diffusion path, i.e. a collection of points of the trajectory of $Y$, including the (given) starting point at time 0 and the ending point at time $T$. We call it the skeleton of the process and we represent it as

$$
\S_{1}:=\left\{\left(t_{0}, y_{0}\right),\left(t_{1}, y_{1}\right), \ldots,\left(t_{m}, y_{m}\right)\right\}
$$

where $0=t_{0}<t_{1}<\cdots<t_{m}=T$. Before stating the relevant results on EA1, we fix some preliminary notation. Let $\mathrm{W}^{(s, x ; t, y)}$ denote the probability measure of a Brownian bridge starting at time $s$ at location $x$ and finishing at time $t$ at location $y$. We also introduce the following representation of the exit times: for any measurable set $B \subset \mathbb{R}$,

$$
\tau_{B}:=\inf \left\{t \in[0, T]: \omega_{t} \notin B\right\},
$$

under the convention $\inf \{\varnothing\}=+\infty$. We will denote by $q^{\mathrm{W}}\left(s, x ; t, y ; l_{1}, l_{2}\right)$ the exit probability of the $(s, x) \rightarrow(t, y)$ Brownian bridge from a given set $\left(l_{1}, l_{2}\right)$ under the condition $x, y \in$ $\left(l_{1}, l_{2}\right)$ :

$$
q^{\mathrm{W}}\left(s, x ; t, y ; l_{1}, l_{2}\right):=\mathrm{W}^{(s, x ; t, y)}\left(\tau_{\left(l_{1}, l_{2}\right)} \leq T \mid x, y \in\left(l_{1}, l_{2}\right)\right) .
$$

\subsection{EA1 results}

Theorem 1, below, brings together the relevant results from Beskos et al. (2006a). It states the conditions on (6) that allow the application of EA1 and it characterises the conditional law of the process $Y$ given the skeleton.

Theorem 1. Under conditions (BO)-(B2) we can apply EA1 to simulate a skeleton (8) of the process $Y$. For any event $B \in \mathcal{C}$,

$$
\mathrm{Q}(B)=\mathrm{E}_{S_{1}}\left[\mathrm{~W}^{S_{1}}(B)\right],
$$

where $\mathrm{W}^{S_{1}}$ denotes the product measure: $\mathrm{W}^{S_{1}}:=\bigotimes_{i=1}^{m} \mathrm{~W}^{\left(t_{i-1}, y_{i-1} ; t_{i}, y_{i}\right)}$. 
Therefore, the conditional law of the process $Y$, given the skeleton, is the product law of Brownian bridges connecting the points of the skeleton. This implies that, by conditioning on $\varsigma_{1}$, we reduce the problem of the simulation from an unknown probability measure $\mathrm{Q}$ to the problem of the simulation from Brownian bridge measures. The following result provides a simple application of this construction.

Corollary 1. Let $\varsigma_{1}$ be the skeleton of $Y$ generated by EA1. Then, for any $l_{1}<y_{0}<l_{2}$,

$$
\mathrm{Q}\left(\tau_{\left(l_{1}, l_{2}\right)}>T\right)=\mathrm{E}_{\delta_{1}}\left[\prod_{i=1}^{m}\left(1-q^{\mathrm{W}}\left(t_{i-1}, y_{i-1} ; t_{i}, y_{i} ; l_{1}, l_{2}\right)\right) \mathbf{1}_{\left\{y_{i} \in\left(l_{1}, l_{2}\right)\right\}}\right] .
$$

Proof. For any $i=1,2, \ldots, m$, let $C_{i}$ denote the set of continuous functions on $\left[t_{i-1}, t_{i}\right]$ and let $\mathcal{C}_{i}$ denote the corresponding $\sigma$-algebra. We define the following events:

$$
B_{i}:=\left\{\omega_{t} \in\left(l_{1}, l_{2}\right) ; t_{i-1} \leq t \leq t_{i}\right\} \in \mathcal{C}_{i} .
$$

Then, $\left\{\tau_{\left(l_{1}, l_{2}\right)}>T\right\}=B_{1} \times B_{2} \times \cdots \times B_{m}$, so that, from (10) and the definition of $\mathrm{W}^{S_{1}}$,

$$
\begin{aligned}
\mathrm{Q}\left(\tau_{\left(l_{1}, l_{2}\right)}>T\right) & =\mathrm{E}_{\delta_{1}}\left[\prod_{i=1}^{m} \mathrm{~W}^{\left(t_{i-1}, y_{i-1} ; t_{i}, y_{i}\right)}\left(B_{i}\right)\right] \\
& =\mathrm{E}_{\delta_{1}}\left[\prod_{i=1}^{m}\left(1-q^{\mathrm{W}}\left(t_{i-1}, y_{i-1} ; t_{i}, y_{i} ; l_{1}, l_{2}\right)\right) \mathbf{1}_{\left\{y_{i} \in\left(l_{1}, l_{2}\right)\right\}}\right] .
\end{aligned}
$$

\subsection{Crossing probability of the Brownian bridge}

2.4.1. One-sided crossing probability. We recall the problem of the evaluation of the crossing probability (9) for Brownian motion. Let us assume that $l_{1}=-\infty$. It turns out that, for any $l_{2} \in \mathbb{R}$,

$$
q^{\mathrm{W}}\left(s, x ; t, y ;-\infty, l_{2}\right)=\exp \left(-2 \frac{\left(l_{2}-y\right)\left(l_{2}-x\right)}{t-s}\right) .
$$

An analogous result holds for the lower barrier case, $l_{2}=+\infty$. Expression (11) can be derived easily from the Bachelier-Levy construction, which provides an explicit formula for the crossing probability of a slope boundary for the Brownian motion (see, e.g. Lerche (1986)).

2.4.2. Two-sided crossing probability. The problem of determining the two-sided crossing probability of the Brownian bridge is more challenging than the one-sided problem. Although it has been extensively studied in the literature (see, e.g. Bertoin and Pitman (1994)), a closed-form expression is not available. In fact, available representations are given in terms of an infinite sum. Nevertheless, here we state a convergence result (Proposition 1, below) which will allow us to construct a suitable Monte Carlo algorithm for the double barrier case. Our approach relies on classical results of Doob (1949) and Anderson (1960). For a recent reference, see also Pötzelberger and Wang (2001). In Appendix A, while proving Proposition 1, we will give a brief account of these constructions. Before stating the proposition, we need some additional notation. For any $j \in \mathbb{N}$, we introduce the two functions

$$
\begin{aligned}
P_{j}\left(s, x ; t, y ; l_{1}, l_{2}\right) & :=p_{j}\left(s, x ; t, y ; l_{2}-l_{1}, l_{1}\right)+p_{j}\left(s, x ; t, y ; l_{2}-l_{1}, l_{2}\right), \\
Q_{j}\left(s, x ; t, y ; l_{1}, l_{2}\right) & :=q_{j}\left(s, x ; t, y ; l_{2}-l_{1}, l_{1}\right)+q_{j}\left(s, x ; t, y ; l_{2}-l_{1}, l_{2}\right),
\end{aligned}
$$


where

$$
\begin{aligned}
& p_{j}(s, x ; t, y ; \delta, l)=\exp \left(-\frac{2}{t-s}\left(j \delta+\left(l-x_{1}\right)\right)(j \delta+(l-y))\right), \\
& q_{j}(s, x ; t, y ; \delta, l)=\exp \left(-\frac{2 j}{t-s}\left(j \delta^{2}-\delta(l-x)\right)\right) .
\end{aligned}
$$

Furthermore, we need the following two sequences of real numbers:

$$
\begin{aligned}
& \underline{n}_{k}\left(s, x ; t, y ; l_{1}, l_{2}\right)=\sum_{j=1}^{k}\left(P_{j}\left(s, x ; t, y ; l_{1}, l_{2}\right)-Q_{j}\left(s, x ; t, y ; l_{1}, l_{2}\right)\right), \\
& \bar{n}_{k}\left(s, x ; t, y ; l_{1}, l_{2}\right)=\underline{n}_{k-1}\left(s, x ; t, y ; l_{1}, l_{2}\right)+P_{k}\left(s, x ; t, y ; l_{1}, l_{2}\right) .
\end{aligned}
$$

Proposition 1. For any $-\infty<l_{1}<l_{2}<+\infty$, as $k \rightarrow \infty$,

$$
\begin{aligned}
& \underline{n}_{k}\left(s, x ; t, y ; l_{1}, l_{2}\right) \uparrow q^{\mathrm{W}}\left(s, x ; t, y ; l_{1}, l_{2}\right), \\
& \bar{n}_{k}\left(s, x ; t, y ; l_{1}, l_{2}\right) \downarrow q^{\mathrm{W}}\left(s, x ; t, y ; l_{1}, l_{2}\right) .
\end{aligned}
$$

Proof. See Appendix A.

This result suggests that we can construct two sequences $\left\{\underline{n}_{k}\right\}$ and $\left\{\bar{n}_{k}\right\}$ converging from below and from above, respectively, to the (unknown) probability $q^{\mathrm{W}}$. Crucially, given the parameters of the Brownian bridge $(s, x, t, y)$ and the values of the barriers $\left(l_{1}, l_{2}\right), \underline{n}_{k}$ and $\bar{n}_{k}$ can be evaluated easily for each $k \in \mathbb{N}$.

\section{The exact Monte Carlo algorithm}

\subsection{General setting}

After setting up the theoretical framework, we turn to the description of the Monte Carlo algorithm. In the first place we note that, since the function $\eta(5)$ is monotone increasing, we can express expectation (2) under the measure $\mathrm{Q}$ of the transformed process $Y$ as

$$
v=\mathrm{E}_{\mathrm{Q}}\left[h^{\prime}\left(\omega_{T}\right) \mathbf{1}_{\left\{\tau_{H^{\prime}}>T\right\}}\right],
$$

where $h^{\prime}(\cdot)=h\left(\eta^{-1}(\cdot)\right), H^{\prime}:=\left(a^{\prime}, b^{\prime}\right), a^{\prime}=\eta(a)$, and $b^{\prime}=\eta(b)$. Assuming that $X \in \mathscr{D}$, Theorem 1 ensures that we can apply EA1 to simulate a skeleton $\varsigma_{1}(8)$ of $Y$. Our simulation strategy will then consist of three main steps.

Step 1. Simulate a skeleton $\wp_{1}$ of $Y$ (EA1).

Step 2. Given $\S_{1}$, simulate an unbiased estimator of $v$ in (2).

Step 3. Simulate the Monte Carlo estimator by repeating and averaging.

Under the measure $\mathrm{Q}$, we can define the two unbiased estimators of $v$,

$$
\begin{aligned}
\phi & :=\phi(\omega)=h^{\prime}\left(\omega_{T}\right) \mathbf{1}_{\left\{\tau_{H^{\prime}}>T\right\}} \quad \text { (plain vanilla), } \\
\psi & :=\psi\left(\delta_{1}\right)=\mathrm{E}_{\mathrm{Q}}\left[\phi \mid \varsigma_{1}\right] \quad \text { (Rao-Blackwellised), }
\end{aligned}
$$

generating the Monte Carlo estimators:

$$
\tilde{v}=\frac{\sum_{j=1}^{N} \phi^{(j)}}{N} \quad \text { and } \hat{v}=\frac{\sum_{j=1}^{N} \psi^{(j)}}{N},
$$


where $\left\{\phi^{(j)}\right\}_{j=1,2, \ldots, N}$ and $\left\{\psi^{(j)}\right\}_{j=1,2, \ldots, N}$ are sequences of i.i.d. copies of (17) and (18). We will focus on the problem of the simulation of $\tilde{v}$ and $\hat{v}$ (step 2) given the skeleton. Instead, for the simulation of the skeleton and the issues related to the implementation of EA1 (step 2), we refer the reader to Beskos et al. (2006a).

\subsection{Plain vanilla Monte Carlo estimator}

We recall that, by construction, the skeleton (8) provides a realization of $\omega_{T}$ under $\mathrm{Q}$, namely $\omega_{T} \equiv y_{m}$. Therefore, after generating the skeleton, the simulation of $\phi$ requires only the simulation of the indicator variable in (17) according to

$$
\begin{aligned}
\mathrm{Q}\left(\mathbf{1}_{\left\{\tau_{H^{\prime}}>T\right\}}=1 \mid \wp_{1}\right) & =\mathrm{Q}\left(\tau_{H^{\prime}}>T \mid \wp_{1}\right) \\
& =\prod_{i=1}^{m}\left(1-q^{\mathrm{W}}\left(t_{i-1}, y_{i-1} ; t_{i}, y_{i} ; a^{\prime}, b^{\prime}\right)\right) \mathbf{1}_{\left\{y_{i} \in H^{\prime}\right\}},
\end{aligned}
$$

where the second equality follows from Corollary 1. Simulation from (19) involves the generation of independent events, say $\left\{E_{i}\right\}_{i=1,2, \ldots, m}$, of probabilities

$$
\left\{q^{\mathrm{W}}\left(t_{i-1}, y_{i-1} ; t_{i}, y_{i} ; a^{\prime}, b^{\prime}\right)\right\}_{i=1,2, \ldots, m} .
$$

These events are in fact the crossing events of the Brownian bridges selected by $\varsigma_{1}$. If any $E_{i}$ occurs, we set $\phi \equiv 0$, otherwise $\phi \equiv h^{\prime}\left(y_{m}\right)$. The basic structure of the algorithm is outlined in Algorithm 1.

Algorithm 1. (Plain vanilla Monte Carlo algorithm.)

1. Call EA1 and simulate the skeleton $\wp_{1}=\left\{\left(t_{0}, y_{0}\right),\left(t_{1}, y_{1}\right), \ldots,\left(t_{m}, y_{m}\right)\right\}$.

2. Evaluate $\ell\left(\wp_{1}\right)=\prod_{1}^{m} \mathbf{1}_{\left\{y_{i} \in H^{\prime}\right\}}$. If $\ell\left(\oiint_{1}\right)=0$, go to 5. Otherwise, go to 3 .

3. Set $i=1$.

3.1. Simulate the event $E_{i}$ with probability $q^{\mathrm{W}}\left(t_{i-1}, y_{i-1} ; t_{i}, y_{i} ; a^{\prime}, b^{\prime}\right)$. If $\mathbf{1}_{\left\{E_{i}\right\}}=1$, go to 5. Else if $i=m$, go to 4 .

3.2. Set $i=i+1$ and go to 3.1 .

4. Output $\phi=h^{\prime}\left(y_{m}\right)$.

5. Output $\phi=0$.

6. Repeat 1-5 a sufficiently large number $N$ of times and output

$$
\hat{v}=\left(\frac{1}{N}\right) \sum_{j=1}^{N} \phi^{(j)} .
$$

In the single barrier case the procedure is straightforward since we have a closed-form expression (11) for the crossing probability of the Brownian bridge. In the double barrier case crossing probabilities are not available in closed form. Therefore, in order to generate the crossing events, we will resort to an iterative algorithm which exploits the result of Proposition 1. Our simulation method resembles the convergence series method described in Devroye (1986, p. 156). The steps of the procedure are reported in the pseudo-code of Algorithm 2, below, 
where we have adopted the following convenient notation:

$$
\begin{aligned}
& \underline{n}_{i, k}:=\underline{n}_{k}\left(t_{i-1}, y_{i-1} ; t_{i}, y_{i} ; a^{\prime}, b^{\prime}\right), \\
& \bar{n}_{i, k}:=\bar{n}_{k}\left(t_{i-1}, y_{i-1} ; t_{i}, y_{i} ; a^{\prime}, b^{\prime}\right), \quad i=1,2, \ldots, m, k \in \mathbb{N} .
\end{aligned}
$$

Algorithm 2. (Subroutine to simulate the indicator variable $\mathbf{1}_{\left\{E_{i}\right\}}$ (double barrier case).)

3.1.1 Sample $U \sim$ Unif[0,1]. Set $k=1$.

3.1.2 Evaluate $\underline{n}_{i, k}$ and $\bar{n}_{i, k}$. If $U>\bar{n}_{i, k}$, go to 3.1.3. If $U<\underline{n}_{i, k}$, go to 3.1.4. Else set $k=k+1$ and repeat 3.1.2.

3.1.3 Set $k=\mathcal{N}_{i}$ and output $\mathbf{1}_{\left\{E_{i}\right\}}=0$.

3.1.4 Set $k=\mathcal{N}_{i}$ and output $\mathbf{1}_{\left\{E_{i}\right\}}=1$.

Clearly, for any $i=1,2, \ldots, m$, the efficiency of the sampling scheme in Algorithm 2 is strictly connected to the behaviour of the random variable $\mathcal{N}_{i}$, representing the number of times we need to repeat the control until a decision is taken. Proposition 2, below, guarantees that, for any $i=1,2, \ldots, m, \mathcal{N}_{i}$ has finite moments of every order.

Proposition 2. Let $M_{i}(\alpha)$ be the moment generating function of $\mathcal{N}_{i}$. Then, for any $i=$ $1,2, \ldots, m$, there exists $\varepsilon_{i} \in \mathbb{R}$ such that, for any $\alpha \in\left(-\varepsilon_{i},+\varepsilon_{i}\right)$,

$$
M_{i}(\alpha)<\infty
$$

Proof. We prove the proposition for an arbitrary $i \in\{1,2, \ldots, m\}$. For the construction of the algorithm, for any $k=1,2, \ldots$,

$$
\operatorname{Pr}\left(\mathcal{N}_{i}>k\right)=\operatorname{Pr}\left(U \in\left(\underline{n}_{i, k}, \min \left\{1, \bar{n}_{i, k}\right\}\right)\right) \leq \bar{n}_{i, k}-\underline{n}_{i, k} .
$$

Thus,

$$
\begin{aligned}
M_{i}(\alpha) & =\mathrm{E}\left[\exp \left(\alpha \mathcal{N}_{i}\right)\right] \\
& =\sum_{k=0}^{\infty} \mathrm{e}^{(k+1) \alpha}\left(\operatorname{Pr}\left(\mathcal{N}_{i}>k\right)-\operatorname{Pr}\left(\mathcal{N}_{i}>k+1\right)\right) \\
& \leq \sum_{k=0}^{\infty} \mathrm{e}^{(k+1) \alpha} \operatorname{Pr}\left(\mathcal{N}_{i}>k\right) \\
& =\mathrm{e}^{\alpha} \sum_{k=0}^{\infty} \mathrm{e}^{k \alpha} \operatorname{Pr}\left(\mathcal{N}_{i}>k\right) \\
& =\mathrm{e}^{\alpha}+\mathrm{e}^{\alpha} \sum_{k=1}^{\infty} \mathrm{e}^{k \alpha} \operatorname{Pr}\left(\mathcal{N}_{i}>k\right) \\
& \leq \mathrm{e}^{\alpha}+\mathrm{e}^{\alpha} \sum_{k=1}^{\infty} \mathrm{e}^{k \alpha}\left(\bar{n}_{i, k}-\underline{n}_{i, k}\right)
\end{aligned}
$$


The moment generating function $M_{i}(\alpha)$ is finite for those values of $\alpha$ for which it converges to the infinite series:

$$
\begin{aligned}
\sum_{k=1}^{\infty} \mathrm{e}^{k \alpha}\left(\bar{n}_{i, k}-\underline{n}_{i, k}\right)= & \sum_{k=1}^{\infty} \exp \left(-\frac{2 k}{\Delta_{i}}\left(k \delta_{i}^{2}+\delta_{i}\left(b^{\prime}-x_{2}\right)-\delta_{i}\left(b^{\prime}-x_{1}\right)-\frac{\Delta_{i}}{2} \alpha\right)\right) \\
& +\sum_{k=1}^{\infty} \exp \left(-\frac{2 k}{\Delta_{i}}\left(k \delta_{i}^{2}-\delta_{i}\left(a^{\prime}-S_{i}\right)+\delta\left(a^{\prime}-S_{i-1}\right)-\frac{\Delta_{i}}{2} \alpha\right)\right),
\end{aligned}
$$

where $\Delta_{i}=t_{i}-t_{i-1}$. It is now clear that it exists a neighbourhood $\left(-\varepsilon_{i},+\varepsilon_{i}\right)$ of 0 such that, if $\alpha \in\left(-\varepsilon_{i},+\varepsilon_{i}\right)$, the two series on the right-hand side converge; that is, $M_{i}(\alpha)$ is finite in $\left(-\varepsilon_{i},+\varepsilon_{i}\right)$.

It is worth remarking that in practice the algorithm performs surprisingly well. In fact, for any $i=1,2, \ldots, m, \mathcal{N}_{i}$ is typically very small since the two sequences $\left\{\underline{n}_{k}\right\}_{k=1,2, \ldots}$ and $\left\{\bar{n}_{k}\right\}_{k=1,2, \ldots}$ converge to $q^{\mathrm{W}}$ faster than exponentially. In the numerical example we will present in Section 5 we have verified that in most cases the algorithm comes to a decision after one or two iterations.

\subsection{Rao-Blackwellised Monte Carlo estimator}

Expanding the conditional expectation in (18), we obtain

$$
\psi=h^{\prime}\left(y_{m}\right) \prod_{i=1}^{m}\left(1-q^{\mathrm{W}}\left(t_{i-1}, y_{i-1} ; t_{i}, y_{i} ; a^{\prime}, b^{\prime}\right)\right) \mathbf{1}_{\left\{y_{i} \in H^{\prime}\right\}} .
$$

Expression (20) shows that the simulation of $\psi$ requires the analytic evaluation of the crossing probabilities of Brownian bridges. As we are aware, this is possible only in the single barrier case. So, assuming, for example, an upper barrier $\left(a^{\prime}=-\infty\right)$, from (11) we obtain

$$
\psi=\psi\left(\wp_{1}\right)=h^{\prime}\left(y_{m}\right)\left(\prod_{i=1}^{m}\left(1-\exp \left(-2 \frac{\left(b^{\prime}-y_{i}\right)\left(b^{\prime}-y_{i-1}\right)}{t_{i}-t_{i-1}}\right)\right) \mathbf{1}_{\left\{y_{i} \in\left(-\infty, b^{\prime}\right)\right\}}\right) .
$$

In this context the simulation of $\psi$ requires only the simulation of $\varsigma_{1}$ and the evaluation of expression (21). Repeating the procedure and averaging gives an estimate of $\hat{v}$. The final algorithm (Algorithm 3, below) turns out to be very simple. Unfortunately, in the double barrier case Rao-Blackwellisation is not feasible, since we are not able to evaluate the crossing probabilities in (20) analytically.

Algorithm 3. (Rao-Blackwellised Monte Carlo Algorithm (upper barrier).)

1. Call EAl and simulate the skeleton $\varsigma_{1}$.

2. Evaluate $\psi=\psi\left(\wp_{1}\right)$ according to $(21)$.

3. Repeat 1-2 a sufficiently a large number $N$ of times and output

$$
\hat{v}=\left(\frac{1}{N}\right) \sum_{j=1}^{N} \psi^{(j)}
$$


We point out that, if available, the Rao-Blackwellised estimator is preferable to the plain vanilla. In fact, by Jensen's inequality,

$$
\operatorname{var}(\psi) \leq \operatorname{var}(\phi)
$$

which implies that, under a quadratic loss function, for fixed $N, \hat{v}$ is more efficient than $\tilde{v}$. Furthermore, the simulation of $\hat{v}$ is computationally less demanding than the simulation of $\tilde{v}$. In fact, at each iteration of the Monte Carlo algorithm, the simulation of $\psi$ involves only the simulation of the skeleton, while the simulation of $\phi$ involves the simulation of the skeleton and the simulation of $\phi$ from $\mathrm{Q}_{\mid s_{1}}$.

\section{More general constructions}

\subsection{Exact Monte Carlo via a truncation of the drift}

We now consider the problem of the Monte Carlo estimation of $v$ (16) given a family of diffusion processes (6) satisfying (B0) and the following two conditions.

(B1*) The drift function $\alpha$ is differentiable on the closure $\bar{H}^{\prime}$ of $H^{\prime}$.

(B2*) The function $\left(\alpha^{2}+\alpha^{\prime}\right) / 2$ is bounded on $\bar{H}^{\prime}$.

Under the conditions above, by truncating the drift function $\alpha$ at the barriers $a^{\prime}$ and $b^{\prime}$ and using smoothing techniques, it is possible to define a process $\tilde{Y}$ :

$$
\mathrm{d} \tilde{Y}_{t}=\tilde{\alpha}\left(\tilde{Y}_{t}\right) \mathrm{d} t+\mathrm{d} W_{t}, \quad \tilde{Y}_{0}=y_{0},
$$

that satisfies the desirable conditions (BO)-(B2) (Section 2.1) and such that, for any $u \in H^{\prime}$, $\tilde{\alpha}(u) \equiv \alpha(u)$. We denote by $\tilde{\mathrm{Q}}$ the measure induced by the process $\tilde{Y}$ on the measurable space $(C, \mathcal{C})$.

Theorem 2. Let us consider the two processes $Y$ and $\tilde{Y}$ with SDEs (6) and (22). If $\alpha \equiv \tilde{\alpha}$ for each $u \in H^{\prime}$ and the function $\phi: C \rightarrow \mathbb{R}$ is measurable with respect to $\mathcal{C}_{T \wedge \tau_{H^{\prime}}}$ then

$$
\mathrm{E}_{\mathrm{Q}}[\phi(\omega)]=\mathrm{E}_{\tilde{\mathrm{Q}}}[\phi(\omega)]
$$

Proof. By Girsanov's theorem, the Radon-Nikodym derivative of Q with respect to $\tilde{Q}$ is given by

$$
M_{T}(\omega)=\frac{\mathrm{d} \mathrm{Q}}{\mathrm{d} \tilde{\mathrm{Q}}}(\omega)=\frac{\exp \left(\int_{0}^{T} \alpha\left(\omega_{s}\right) \mathrm{d} \omega_{s}-(1 / 2) \int_{0}^{T} \alpha^{2}\left(\omega_{s}\right) \mathrm{d} s\right)}{\exp \left(\int_{0}^{T} \tilde{\alpha}\left(\omega_{s}\right) \mathrm{d} \omega_{s}-(1 / 2) \int_{0}^{T} \tilde{\alpha}^{2}\left(\omega_{s}\right) \mathrm{d} s\right)} .
$$

By the measurability assumption on $\phi$ and the martingale property of $\left\{M_{t}, \mathcal{C}_{t}\right\}_{0 \leq t \leq T}$, we have

$$
\begin{aligned}
\mathrm{E}_{\mathrm{Q}}[\phi(\omega)] & =\mathrm{E}_{\tilde{\mathrm{Q}}}\left[\phi(\omega) M_{T}(\omega)\right] \\
& =\mathrm{E}_{\tilde{\mathrm{Q}}}\left[\mathrm{E}_{\tilde{\mathrm{Q}}}\left[\phi(\omega) M_{T}(\omega) \mid \mathcal{C}_{T \wedge \tau_{H^{\prime}}}\right]\right] \\
& =\mathrm{E}_{\tilde{\mathrm{Q}}}\left[\phi(\omega) \mathrm{E}_{\tilde{\mathrm{Q}}}\left[M_{T}(\omega) \mid \mathcal{C}_{T \wedge \tau_{H^{\prime}}}\right]\right] \\
& =\mathrm{E}_{\tilde{\mathrm{Q}}}\left[\phi(\omega) M_{T \wedge \tau_{H^{\prime}}}\right] .
\end{aligned}
$$

Since on the interval $\left[0, T \wedge \tau_{H^{\prime}}\right)$ the two drift functions $\alpha$ and $\tilde{\alpha}$ coincide, it turns out that $M_{T \wedge \tau_{H^{\prime}}}=1$, Q -almost surely . The conclusion then follows. 
In our framework, since $\phi(\omega)=h^{\prime}\left(\omega_{T}\right) \mathbf{1}_{\left\{\tau_{H^{\prime}}>T\right\}}$ is clearly $\mathcal{C}_{T \wedge \tau_{H^{\prime}}}$-measurable, Theorem 2 implies that

$$
v=\mathrm{E}_{\mathrm{Q}}\left[h^{\prime}\left(\omega_{T}\right) \mathbf{1}_{\left\{\tau_{H^{\prime}}>T\right\}}\right]=\mathrm{E}_{\tilde{Q}}\left[h^{\prime}\left(\omega_{T}\right) \mathbf{1}_{\left\{\tau_{H^{\prime}}>T\right\}}\right] .
$$

Therefore, we can simulate an unbiased estimator of $v$ by applying the exact Monte Carlo method to the auxiliary process $\tilde{Y}$ (satisfying conditions (BO)-(B2)). This strategy is particularly powerful when we deal with a double barrier problem. In fact, in that case $H^{\prime}$ is a bounded interval and we just need to ensure that the drift $\alpha$ 'behaves well' in a compact set $\bar{H}^{\prime}$. This is naturally satisfied by most models. However, in the single barrier case we should still assume boundedness of $\alpha$ on one side (either $\left(-\infty, b^{\prime}\right]$ or $\left[a^{\prime},+\infty\right)$ ).

\subsection{Exact Monte Carlo via EA2}

We replace condition (B2) with the following less restrictive condition:

(B2**) For all $u \in \mathbb{R},\left(\alpha^{2}+\alpha^{\prime}\right) / 2$ is bounded below and it is bounded above either on $(-\infty, u)$ or on $(u,+\infty)$.

We assume, without loss of generality, that the function $\left(\alpha^{2}+\alpha^{\prime}\right) / 2$ is bounded on the intervals $\{(u,+\infty)\}_{u \in \mathbb{R}}$. EA2 was introduced in Beskos et al. (2006a). A crucial difference between it and EA1 lies in the fact that it outputs a richer structure than $\wp_{1}(8)$. In fact, in addition to the starting point $\left(0, y_{0}\right)$ and the ending point $\left(T, y_{T}\right)$ of the diffusion path, it also includes its minimum and the time at which this minimum is achieved, say $\left(t^{*}, y^{*}\right)$. It is convenient to choose a representation of $\delta_{2}$ which takes into account this underlying structure:

$$
s_{2}:=\left\{\left(t_{0}, y_{0}\right),\left(t_{1}, y_{1}\right), \ldots,\left(t_{m_{1}}, y_{m_{1}}\right), \ldots,\left(t_{m_{2}}, y_{m_{2}}\right)\right\},
$$

where $0=t_{0}<t_{1}<\cdots<t_{m_{1}}=t^{*}<\cdots<t_{m_{2}}=T$, so that the minimum can be easily identified as $\left(t_{m_{1}}, y_{m_{1}}\right) \equiv\left(t^{*}, y^{*}\right)$. We denote by $\mathrm{Q}-y^{*}$ the probability measure induced by the process $Y-y^{*}:=\left\{Y_{t}-y^{*}: 0 \leq t \leq T\right\}$ on $(C, \mathcal{C})$ and denote by $\mathrm{W}_{+}^{(s, x ; t, y)}$ the probability measure of a three-dimensional Bessel bridge from $(s, x)$ to $(t, y)$. Furthermore, we will denote crossing probabilities as follows:

$$
q^{\mathrm{W}_{+}}\left(s, x ; t, y ; l_{1}, l_{2}\right):=\mathrm{W}_{+}^{(s, x ; t, y)}\left(\tau_{\left(l_{1}, l_{2}\right)} \leq T \mid x, y \in\left(l_{1}, l_{2}\right)\right) .
$$

The following result is the analogue of Theorem 1 and it can be derived from the construction presented in Beskos et al. (2006a).

Theorem 3. Under conditions $C O, C 1$ and $B 2^{* *}$, we can apply EA2 to simulate a skeleton $\varsigma_{2}$ (23) of the process $Y$. For any event $A \in \mathcal{C}$,

$$
\mathrm{Q}-y^{*}(A)=\mathrm{E}_{\varsigma_{2}}\left[\mathrm{~W}_{+}^{\wp_{2}}(A)\right],
$$

where $\mathrm{W}_{+}^{\S_{2}}=\bigotimes_{i=1}^{m_{2}} \mathrm{~W}_{+}^{\left(t_{i-1}, y_{i-1}-y^{*} ; t_{i}, y_{i}-y^{*}\right)}$.

Theorem 3 justifies the following suitable representation of the crossing probability of the process $Y$.

Corollary 2. Let $\varsigma_{2}$ be the skeleton of $Y$ generated by EA2. Then, for any $l_{1}<y_{0}<l_{2}$,

$$
\mathrm{Q}\left(\tau_{\left(l_{1}, l_{2}\right)}>T\right)=\mathrm{E}_{s_{2}}\left[\mathbf{1}_{\left\{y^{*}>l_{1}\right\}} \prod_{i=1}^{m_{2}}\left(1-q^{\mathrm{W}_{+}}\left(t_{i-1}, y_{i-1}^{*} ; t_{i}, y_{i}^{*} ;-\infty, l_{2}^{*}\right)\right) \mathbf{1}_{\left\{C_{i}\right\}}\right],
$$

where, for any $i=1,2, \ldots, m_{2}, y_{i}^{*}:=y_{i}-y^{*}, l_{2}^{*}:=l_{2}-y^{*}$, and $C_{i}:=\left\{y_{i}<l_{2}\right\}$. 
Proof. From Theorem 3 we derive the following representation:

$$
\mathrm{Q}\left(\tau_{\left(l_{1}, l_{2}\right)}>T\right)=\mathrm{Q}-y^{*}\left(\tau_{\left(l_{1}^{*}, l_{2}^{*}\right)}>T\right)=\mathrm{E}_{\varsigma_{2}}\left[\mathrm{~W}_{+}^{\varsigma_{2}}\left(\tau_{\left(l_{1}^{*}, l_{2}^{*}\right)}>T\right)\right],
$$

where we have set $l_{1}^{*}=l_{1}-y^{*}$. Proceeding as in the proof of Corollary 1 , for any $i=$ $1,2, \ldots, m_{2}$, we define the events

$$
B_{i}^{*}=\left\{\omega_{t} \in\left(l_{1}-y^{*}, l_{2}-y^{*}\right) ; t_{i-1} \leq t \leq t_{i}\right\},
$$

so that $\left\{\tau_{\left(l_{1}-y^{*}, l_{2}-y^{*}\right)}>T\right\}=B_{1}^{*} \times \cdots \times B_{m_{2}}^{*}$. Therefore, by the definition of $\mathrm{W}_{+}^{\ell_{2}}$ we have

$$
\begin{aligned}
& \mathrm{W}_{+}^{\delta_{2}}\left(\tau_{\left(l_{1}-y^{*}, l_{2}-y^{*}\right)}>T\right)=\prod_{i=1}^{m_{2}} \mathrm{~W}_{+}^{\left(t_{i-1}, y_{i-1}^{*} ; t_{i}, y_{i}^{*}\right)}\left(B_{i}^{*}\right) \\
& =\prod_{i=1}^{m_{2}}\left(1-q^{\mathrm{W}_{+}}\left(t_{i-1}, y_{i-1}^{*} ; t_{i}, y_{i}^{*} ; l_{1}^{*}, l_{2}^{*}\right)\right) \mathbf{1}_{\left\{y_{i} \in\left(l_{1}, l_{2}\right)\right\}} \text {. }
\end{aligned}
$$

Now, if $\left\{y^{*} \leq l_{1}\right\} \equiv\left\{y_{m_{1}} \leq l_{1}\right\}$, (25) is clearly null in agreement with (24); if on the other hand $\left\{y^{*}>l_{1}\right\}$, (24) follows from (25) using the positivity of the Bessel process.

Now, substituting appropriately into (24), we obtain the following probability:

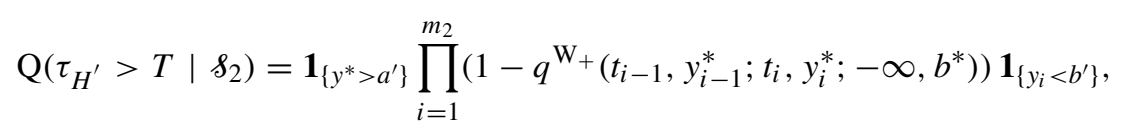

where we have set $b^{*}=b^{\prime}-y^{*}$. Analytic evaluation of (26) is not feasible, since a closed-form formula for the crossing probability $q^{\mathrm{W}_{+}}$of the Bessel bridge is not available. Consequently, we are not able to simulate the Rao-Blackwellised estimator $\psi(18)$ and the corresponding Monte Carlo estimator $\hat{v}$. However, in the lower barrier case $\left(b^{*}=+\infty\right)$,

$$
\mathrm{Q}\left(\tau_{H^{\prime}}>T \mid \wp_{2}\right)=\mathbf{1}_{\left\{y^{*}>a^{\prime}\right\}},
$$

so that the simulation of the plain vanilla estimator $\phi(17)$ is immediate. On the other hand, when $b^{*}$ is finite (either in the upper barrier or in the double barrier case), the simulation of $\phi$ involves the generation of events of probabilities $\left\{q^{\mathrm{W}_{+}}\left(t_{i-1}, y_{i-1}^{*} ; t_{i}, y_{i}^{*} ;-\infty, b^{*}\right)\right\}_{i=1,2, \ldots, m_{2}}$. To this end, we have defined an appropriate algorithm, similar in spirit to Algorithm 2. The algorithm is based on the following result. For any $k \in \mathbb{N}$, let

$$
\begin{aligned}
& \bar{n}_{k}^{*}(s, x ; t, y ; l)=\frac{1-\underline{n}_{k}(s, x ; t, y ; 0, l)}{1-\exp (-2 x y /(t-s))}, \\
& \underline{n}_{k}^{*}(s, x ; t, y ; l)=\frac{1-\bar{n}_{k}(s, x ; t, y ; 0, l)}{1-\exp (-2 x y /(t-s))},
\end{aligned}
$$

where $\left\{\underline{n}_{k}\right\}$ and $\left\{\bar{n}_{k}\right\}$ are respectively defined by (12) and (13).

Proposition 3. For any $b^{*}>0$ and $i \in\left\{1,2, \ldots, m_{2}\right\}$, as $k \rightarrow+\infty$,

$$
\begin{aligned}
& \underline{n}_{k}^{*}\left(t_{i-1}, y_{i-1}^{*} ; t_{i}, y_{i}^{*} ; b^{*}\right) \uparrow 1-q^{\mathrm{W}_{+}}\left(t_{i-1}, y_{i-1}^{*} ; t_{i}, y_{i}^{*} ;-\infty, b^{*}\right), \\
& \bar{n}_{k}^{*}\left(t_{i-1}, y_{i-1}^{*} ; t_{i}, y_{i}^{*} ; b^{*}\right) \downarrow 1-q^{\mathrm{W}_{+}}\left(t_{i-1}, y_{i-1}^{*} ; t_{i}, y_{i}^{*} ;-\infty, b^{*}\right) .
\end{aligned}
$$


Proof. For any $l^{*}>0$ and $i \in\left\{1,2, \ldots, m_{2}\right\}$, using the representation of a Bessel bridge as a Brownian bridge conditioned to be positive,

$$
1-q^{\mathrm{W}_{+}}\left(t_{i-1}, y_{i-1}^{*} ; t_{i}, y_{i}^{*} ;-\infty, l^{*}\right)=\frac{1-q^{\mathrm{W}}\left(t_{i-1}, y_{i-1}^{*} ; t_{i}, y_{i}^{*} ; 0, l^{*}\right)}{1-q^{\mathrm{W}}\left(t_{i-1}, y_{i-1}^{*} ; t_{i}, y_{i}^{*} ; 0, \infty\right)},
$$

whereas, by the Bachelier-Levy formula,

$$
1-q^{\mathrm{W}}\left(t_{i-1}, y_{i-1}^{*} ; t_{i}, y_{i}^{*} ; 0, \infty\right)=1-\exp \left(-2 \frac{y_{i-1}^{*} y_{i}^{*}}{t_{i}-t_{i-1}}\right) .
$$

Furthermore, as a consequence of (14) and (15), as $k \rightarrow+\infty$,

$$
\begin{aligned}
& 1-\underline{n}_{k}\left(t_{i-1}, y_{i-1}^{*} ; t_{i}, y_{i}^{*} ; 0, l^{*}\right) \downarrow 1-q^{\mathrm{W}}\left(t_{i-1}, y_{i-1}^{*} ; t_{i}, y_{i}^{*} ; 0, l^{*}\right), \\
& 1-\bar{n}_{k}\left(t_{i-1}, y_{i-1}^{*} ; t_{i}, y_{i}^{*} ; 0, l^{*}\right) \uparrow 1-q^{\mathrm{W}}\left(t_{i-1}, y_{i-1}^{*} ; t_{i}, y_{i}^{*} ; 0, l^{*}\right),
\end{aligned}
$$

so that by combining (27) and (28), the conclusion follows.

The simulation from probabilities $q^{\mathrm{W}_{+}}$in (26) is analogous to the simulation from $q^{\mathrm{W}}$ in the double barrier case, as described in Algorithm 2. Namely, we simulate a [0,1]-uniformly distributed random variable and we compare it stepwise with couples of values $\left(\underline{n}_{k}^{*}, \bar{n}_{k}^{*}\right)$ ( $k$ increasing) until a decision is taken. In this way we can simulate the crossing event from (26) and, consequently, the plain vanilla estimator (17).

\section{Numerical example}

To test our algorithm, we consider the following model:

$$
\mathrm{d} Y_{t}=\sin \left(Y_{t}\right) \mathrm{d} t+\mathrm{d} W_{t}, \quad Y_{0}=y_{0}, 0 \leq t \leq T .
$$

It is easy to verify that $Y$ satisfies conditions (B0)-(B2) for the exact Monte Carlo algorithm. Let us suppose that we want to evaluate

$$
v=\mathrm{E}\left[Y_{T} \mathbf{1}_{\{\tau>T\}} \mid Y_{0}=y_{0}\right],
$$

where, as usual, $\tau=\inf \left\{t \geq 0: Y_{t} \notin H\right\}$ with $H=(a, b) \subset \mathbb{R}$ such that $y_{0} \in H$. Since we do not know the law at time $T$ of the killed diffusion, it is clear that the explicit computation of $v$ is not possible and we resort to Monte Carlo methods to estimate $v$. In this simulation study we investigate the performance of the estimator of $v$ produced by the exact Monte Carlo method (hereafter E1).

The plots in Figure 1 show a comparison between E1 and the estimators based on the continuous Euler scheme (E2) and on the discrete Euler scheme (E3). In particular, given a Monte Carlo sample sufficiently large $\left(10^{6}\right)$, for different choices of the starting point $y_{0}$ and the barriers' values $a$ and $b$, we have computed the estimates of E1 (dotted line) and the estimates produced by E2 and E3 for different discretization intervals. Then we have plotted the values of E2 (crosses) and E3 (circles) versus the number of discretization intervals.

As we expected, the values of E2 and E3 converge to E1 as the number of discretization intervals increases. Indeed, it was shown in Gobet (2000) that, for killed diffusions, the weak approximation error of Euler schemes decreases to 0 as the number of discretization intervals increases. When the Monte Carlo sample size is large enough, the Monte Carlo error 


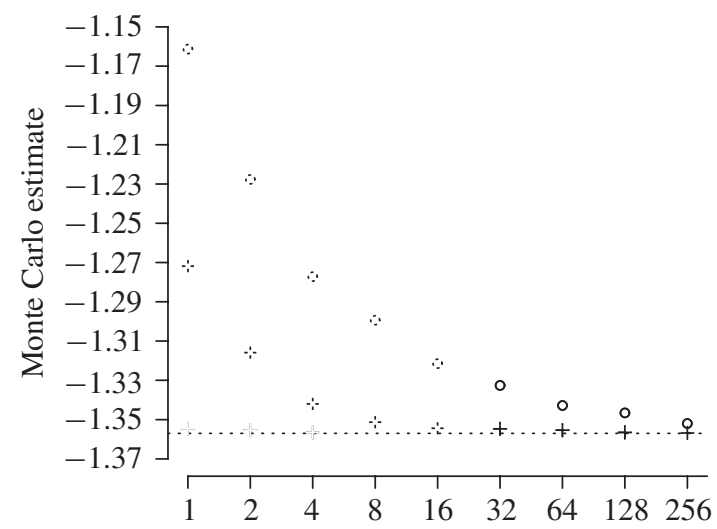

(a)

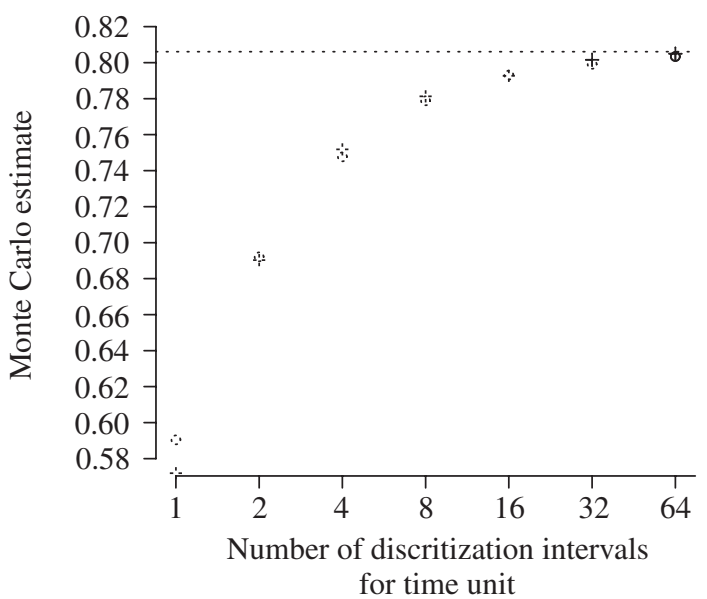

(c)

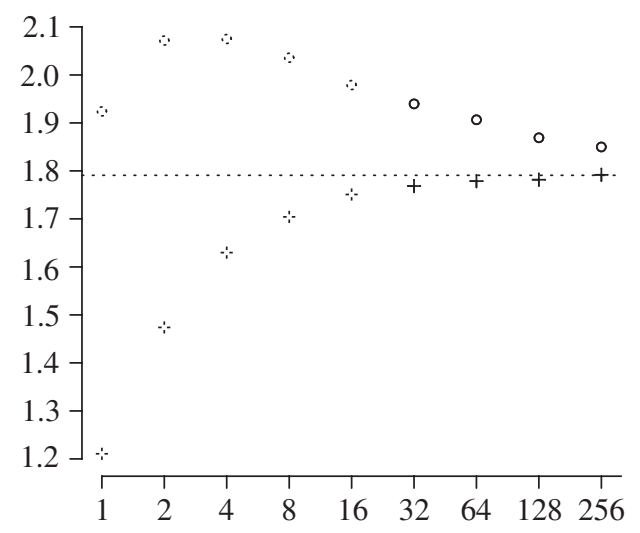

(b)

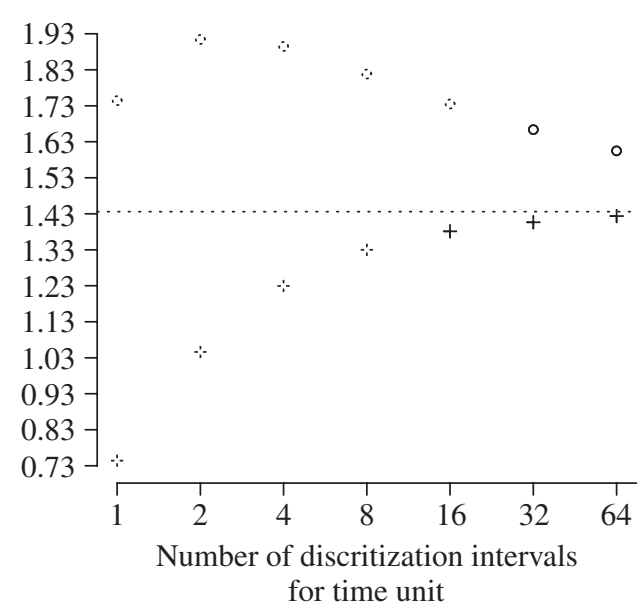

(d)

Figure 1: Monte Carlo estimates of $v$ for the model (29) based on the exact Monte Carlo (dotted line), the discrete Euler scheme (circles), and the continuous Euler scheme (crosses). Monte Carlo estimates based on the Euler schemes are associated with the corresponding number of disretization intervals. The Monte Carlo sample size equals $10^{6}$. (a) $y_{0}=0, b=3$, and $T=5$. (b) $y_{0}=1.5, b=4.5$, and $T=5$. (c) $y_{0}=0, a=-3.5, b=4.5$, and $T=5$. (d) $y_{0}=2, a=1, b=4.5$, and $T=5$

is negligible and the estimated values are affected mainly by the discretization error. In this context the distance between the values of E2 and E3 and the dotted line is a good representation of the (weak) discretization error affecting the Euler schemes, and their convergence to the dotted line reflects the theoretical convergence of the corresponding expected values. Furthermore, according to the conclusions of Gobet, we note that the estimates based on the continuous Euler scheme show better convergence than the estimates based on the discrete Euler scheme.

Comparing the performances of E2 and E3 along the four plots, we observe that both in the single barrier case (Figure 1(a) and (b)) and in the double barrier case (Figure 1(c) and (d)) Euler-based estimators behave very poorly when the starting point of the process is other than 0 (Figure 1(b) and (d)). Moreover, in these cases E2 converges to the E1 value from below while 
E3 converges from above. The reasons why Euler schemes behave in this way deserves further attention from both an empirical and a theoretical point of view.

In practical applications there is an interest in the comparison between the computational times of the exact Monte Carlo method and the Euler-based methods. However, such comparison is not straightforward since Euler schemes are subjected to a trade-off between computational time and discretization error, i.e. as the estimates of E2 and E3 converge to the dotted line, the time needed to produce them increases. In each plot we use symbols made from solid and dashed lines. The solid line symbols represent simulations which required greater computational effort than E1. In these cases our algorithm is both more accurate and more efficient.

\section{Conclusions}

In this paper we have developed and implemented a novel Monte Carlo method for the estimation of the expected value of a class of functionals of diffusion processes. In particular we considered functionals involving barriers. In these cases the estimation of the expected value is typically challenging. In fact, it is common practice to discretize the underlying diffusion and run Monte Carlo simulation on the discretized process. This clearly introduces a bias in Monte Carlo simulation. Such bias can be reduced only at the cost of a larger computational effort. In comparison, our method turns out to be as unbiased and efficient as our simulation study for the sine model demonstrated.

The application of the exact algorithm to the multidimensional case is limited to unit diffusion coefficient SDEs whose drift can be expressed as the gradient of a potential (Langevin-type diffusions) (Beskos et al. (2008)). In this context the exact algorithm returns a skeleton that gives rise to a factorization of the conditional process in terms of the product of multidimensional independent Brownian bridges. For rectangular 'killing' regions, a straightforward multivariate exact Monte Carlo algorithm for the barrier problem can be constructed by simulating the crossing events for each of the components of the Brownian bridges. For more general killing regions, the simulation of crossing events of the Brownian bridges is a challenging and open problem. Interesting proposals in this direction can be found in Lépingle (1995), Gobet (2001), and Bossy et al. (2004) for the related problem of the approximation of a reflected multidimensional diffusion.

The methodology presented in this paper suggests several further developments and ideas for future research.

A major objective is to extend the algorithm to larger classes of diffusion processes as well as to other time-continuous processes such as jump-diffusion processes, Levy processes, or stochastic volatility processes. Some relatively minor extensions of the basic exact Monte Carlo algorithm have been proposed in Section 4. We are currently working on more substantial generalizations of the algorithm including Monte Carlo simulation of jump-diffusion processes with state dependent intensity.

We have concentrated here on the (Monte Carlo) barrier problem. This is especially relevant in option pricing for the evaluation of barrier and lookback options and in credit risk modelling for the evaluation of defaultable derivatives. However, we believe that there is a wide range of other relevant Monte Carlo problems arising in finance to which the exact Monte Carlo framework can be successfully applied. For instance, current work involves Monte Carlo estimation of the Greeks. 
Finally, even though the results from our simulation study are promising, it is worth carrying out a more comprehensive numerical simulation. In particular, the efficiency of our method in comparison with Euler-based methods needs to be investigated in more detail.

\section{Appendix A. Brownian bridge two-sided crossing probability (proof of Proposition 1)}

Let $W$ be the standard Brownian motion and $W^{(s, x ; t, y)}$ be the $(s, x) \rightarrow(t, y)$ Brownian bridge. It is well known that, for any $\delta>0$,

$$
W_{u}^{(0, x ; \delta, y)} \stackrel{\mathrm{D}}{=} x+\frac{u}{\delta}(y-x)+\frac{\delta-u}{\sqrt{\delta}} W_{u /(\delta-u)}, \quad 0 \leq u \leq \delta,
$$

where ' $\stackrel{D}{=}$ ' denotes equality in distribution from which we can derive

$$
p^{\mathrm{W}}\left(s, x ; t, y ; l_{1}, l_{2}\right)=\operatorname{Pr}\left(l_{1}(u)<W_{u}<l_{2}(u), u \geq 0\right),
$$

where we have set

$$
\begin{aligned}
& l_{1}(u):=\frac{l_{1}-y}{\sqrt{t-s}} u+\frac{l_{1}-x}{\sqrt{t-s}}, \\
& l_{2}(u):=\frac{l_{2}-y}{\sqrt{t-s}} u+\frac{l_{2}-x}{\sqrt{t-s}} .
\end{aligned}
$$

Now, under the usual convention that $\inf \{\varnothing\}=\infty$, we define the following stopping times:

$$
\tau^{*}=\inf \left\{u \geq 0: W_{u} \leq l_{1}(u) \text { or } W_{u} \geq l_{2}(u)\right\}
$$

and

$$
\begin{aligned}
& \tau_{j, 1}=\inf \left\{u \geq \tau_{j-1,2}: W_{u} \leq l_{1}(u)\right\}, \\
& \tau_{j, 2}=\inf \left\{u \geq \tau_{j-1,1}: W_{u} \geq l_{2}(u)\right\}, \quad j=1,2, \ldots,
\end{aligned}
$$

under the convention that $\tau_{0,1}=\tau_{0,2}=0$. Let us define the following related events:

$$
\begin{array}{rlrl}
A & :=\left\{\tau^{*}<\infty\right\}, & A_{1} & :=\left\{\tau_{1,1}<\tau_{1,2}\right\}, \\
A_{2} & :=\left\{\tau_{1,2}<\tau_{1,1}\right\}, \quad A_{j, n} & :=\left\{\tau_{j, n}<\infty\right\}, \quad \text { for } j=1,2, \ldots \text { and } n=1,2 .
\end{array}
$$

Using reflecting properties of the Brownian motion, it is possible to show that (see, e.g. Anderson (1960, Theorem 4.1)), for any $j \in \mathbb{N}$,

$$
\begin{aligned}
\operatorname{Pr}\left(A_{2 j-1,2}\right) & =p_{j}\left(s, x ; t, y ; \delta, l_{2}\right), & \operatorname{Pr}\left(A_{2 j-1,1}\right) & =p_{j}\left(s, x ; t, y ; \delta, l_{1}\right), \\
\operatorname{Pr}\left(A_{2 j, 2}\right) & =q_{j}\left(s, x ; t, y ; \delta, l_{2}\right), & \operatorname{Pr}\left(A_{2 j, 1}\right) & =q_{j}\left(s, x ; t, y ; \delta, l_{1}\right),
\end{aligned}
$$

with $\delta=l_{2}-l_{1}$. Straightforward probabilistic arguments lead to the following results:

(i) $\left\{A_{j, 1}\right\} \downarrow \varnothing$ and $\left\{A_{j, 2}\right\} \downarrow \varnothing$,

(ii) $A=A_{2} \sqcup A_{1}=A_{1,2} \cup A_{1,1}$,

(iii) $A_{2}=\bigsqcup_{j=1}^{+\infty}\left(A_{2 j-1,2}-A_{2 j, 2}\right)$ and $A_{1}=\bigsqcup_{j=1}^{+\infty}\left(A_{2 j-1,1}-A_{2 j, 1}\right)$,

(iv) for any $j=1,2, \ldots, A_{j, 2} \cap A_{j, 1}=A_{j+1,2} \cup A_{j+1,1}$, 
where with the symbol $\sqcup$ we denote the disjoint union. Finally, we can write

$$
\begin{aligned}
q^{\mathrm{W}}\left(s, x_{1} ; t, x_{2} ; l_{1}, l_{2}\right) & =\operatorname{Pr}(A) \\
& =\operatorname{Pr}\left(A_{2}\right)+\operatorname{Pr}\left(A_{1}\right) \\
& =\sum_{j=1}^{+\infty}\left(\left(\operatorname{Pr}\left(A_{2 j-1,2}\right)-\operatorname{Pr}\left(A_{2 j, 2}\right)\right)+\left(\operatorname{Pr}\left(A_{2 j-1,1}\right)-\operatorname{Pr}\left(A_{2 j, 1}\right)\right)\right) \\
& =\sum_{j=1}^{+\infty}\left(P_{j}\left(s, x ; t, y ; l_{1}, l_{2}\right)-Q_{j}\left(s, x ; t, y ; l_{1}, l_{2}\right)\right) \\
& =\lim _{k \rightarrow \infty} \underline{n}_{k}\left(s, x ; t, y ; l_{1}, l_{2}\right),
\end{aligned}
$$

where we have used (ii) and (iii). From (i), for any $j=1,2, \ldots$,

$$
\operatorname{Pr}\left(A_{2 j-1,2}\right)-\operatorname{Pr}\left(A_{2 j, 2}\right)+\operatorname{Pr}\left(A_{2 j-1,1}\right)-\operatorname{Pr}\left(A_{2 j, 1}\right) \geq 0,
$$

so that $\left\{\underline{n}_{k}\right\}_{k=1,2, \ldots}$ is increasing, proving (14). On the other side, from (ii) and (iv), the sequence $\left\{\bar{n}_{k}\right\}_{k=1,2, \ldots}$ (13) can be written in the following way:

$$
\begin{aligned}
\bar{n}_{1}\left(s, x ; t, y ; l_{1}, l_{2}\right) & =\operatorname{Pr}\left(A_{1,2} \cup A_{1,1}\right)+\operatorname{Pr}\left(A_{1,2} \cap A_{1,1}\right) \\
& =\operatorname{Pr}(A)+c_{1} \\
& =q^{*}\left(s, x ; t, y ; l_{1}, l_{2}\right)+c_{1}, \\
\bar{n}_{k}\left(s, x ; t, y ; l_{1}, l_{2}\right) & =\bar{n}_{k-1}\left(s, x ; t, y ; l_{1}, l_{2}\right)+\left(c_{k}-c_{k-1}\right) \\
& =\operatorname{Pr}(A)+c_{k} \\
& =q^{\mathrm{W}}\left(s, x ; t, y ; l_{1}, l_{2}\right)+c_{k},
\end{aligned}
$$

where $\left\{c_{k}=\operatorname{Pr}\left(A_{2 k-1,2} \cap A_{2 k-1,1}\right)\right\}_{k=1,2, \ldots}$ is decreasing such that $\lim _{k \rightarrow \infty} c_{k}=0$. Therefore, for any $k=1,2, \ldots$,

$$
q^{\mathrm{W}}\left(s, x ; t, y ; l_{1}, l_{2}\right)+c_{k} \geq q^{\mathrm{W}\left(s, x ; t, y ; l_{1}, l_{2}\right)}+c_{k+1}
$$

and

$$
\lim _{k \rightarrow+\infty} \bar{n}_{k}\left(s, x ; t, y ; l_{1}, l_{2}\right)=\operatorname{Pr}(A)+\lim _{k \rightarrow \infty} c_{k}=\operatorname{Pr}(A)=q^{\mathrm{W}}\left(s, x ; t, y ; l_{1}, l_{2}\right),
$$

so that (15) follows.

\section{Appendix B.}

Let us recall conditions 1-3 of Beskos and Roberts (2005) for the construction of EA1.

(C1) The drift function $\alpha$ is everywhere differentiable.

(C2) $\int_{\mathbb{R}} \exp \left(A(u)-u^{2} / 2 T\right) \mathrm{d} u$ with $A(u)=\int_{0}^{u} \alpha(z) \mathrm{d} z$ is bounded by a constant.

(C3) The function $\left(\alpha^{2}+\alpha^{\prime}\right) / 2$ is bounded.

Conditions (B1)-(B2) in Section 2 trivially imply conditions (C1)-(C3) if the following lemma holds. 
Lemma 1. Suppose that $\alpha$ is a $\mathcal{C}^{1}$ function on $\mathbb{R}$. If $\alpha^{\prime}+\alpha^{2}$ is bounded then so is $\alpha$.

Proof. Suppose to the contrary that either

(i) $\lim \sup _{x \rightarrow+\infty} \alpha(x)=+\infty$ or

(ii) $\liminf x \rightarrow+\infty \alpha(x)=-\infty$

hold, or, alternatively, that (i) or (ii) hold with $x \rightarrow-\infty$. Firstly, suppose that (i) holds. Then there exists a sequence $\left\{x_{i}\right\} \rightarrow+\infty$ with $\left\{\alpha\left(x_{i}\right)\right\} \rightarrow+\infty$. Under the hypothesis of the lemma, for all large enough indices $i$, we have

1. $x_{i}>0$,

2. $\alpha^{\prime}\left(x_{i}\right)<0$,

3. $\alpha\left(x_{i}\right)>\alpha(0)$.

Since the derivative function $\alpha^{\prime}$ is continuous, by the intermediate value theorem, there exists $y_{i} \in\left[0, x_{i}\right]$ such that $\alpha^{\prime}\left(y_{i}\right)=0$ and $\alpha\left(y_{i}\right)>\alpha\left(x_{i}\right)$. Thus, $\left\{\alpha\left(y_{i}\right)\right\} \rightarrow+\infty$ and $\alpha^{\prime}\left(y_{i}\right)+$ $\alpha^{2}\left(y_{i}\right)=\alpha^{2}\left(y_{i}\right)$ is unbounded in $i$ for a contradiction.

Secondly, suppose instead that (ii) holds. Then there exists $\left\{x_{i}\right\} \rightarrow+\infty$ with $\left\{\alpha\left(x_{i}\right)\right\} \rightarrow$ $-\infty$. Then, for all sufficiently large $i$,

1. $x_{i}>0$,

2. $\alpha^{\prime}\left(x_{i}\right)<0$,

3. $\alpha^{\prime}(y)<0$ for all $y>x_{i}$ (otherwise there will be somewhere where $\alpha^{\prime}(y)=0$, leading to a contradiction similar to the one obtained when supposing that (i) holds).

Therefore, for sufficiently large $x, \alpha(x)$ is decreasing so that $\lim _{x \rightarrow+\infty} \alpha(x)=-\infty$. This, combined with the boundedness of the function $\alpha^{\prime}+\alpha^{2}$, implies that, for any $\varepsilon \in(0,1)$, there exists $x_{0}(\varepsilon)$ such that, for any $x \geq x_{0}$, we have

$$
-\left(\frac{1}{\alpha(x)}\right)^{\prime}+\varepsilon=\frac{\alpha^{\prime}(x)}{\alpha^{2}(x)}+\varepsilon \leq 0 .
$$

Choosing $\varepsilon=\frac{1}{2}$ and applying the mean value theorem to the continuous function $\alpha$, we obtain, for any $x \geq x_{0}\left(\frac{1}{2}\right)$ and $y \in(0, x)$,

$$
\frac{1}{\alpha(x)}-\frac{1}{\alpha(y)} \geq \frac{x-y}{2},
$$

leading to a contradiction.

\section{References}

Anderson, T. W. (1960). A modification of the sequential probability ratio test to reduce the sample size. Ann. Math. Statist. 31, 165-197.

Asmussen, S. And Glynn, P. W. (2007). Stochastic Simulation: Algorithms and Analysis. Springer, New York.

Bertoin, J. And Pitman, J. (1994). Path transformations connecting Brownian bridge, excursion and meander. Bull. Sci. Math. 118, 147-166.

Beskos, A. and Roberts, G. O. (2005). Exact simulation of diffusions. Ann. Appl. Prob. 15, 2422-2444.

Beskos, A. and Papaspiliopoulos, O. and Roberts, G. O. (2006a). Retrospective exact simulation of diffusion sample paths with applications. Bernoulli 12, 1077-1098. 
Beskos, A. and Papaspiliopoulos, O. and Roberts, G. O. (2008). A new factorisation of diffusion measure and sample path reconstruction. To appear in Methodology Comput. Appl. Prob..

Beskos, A. and Papaspiliopoulos, O. and Roberts, G. O. and Fearnhead, P. (2006b). Exact and efficient likelihood based inference for discretely observed diffusions (with discussion). J. R. Statist. Soc. B 68, 333-382.

BLACK, F. AND Cox, J. C. (1976). Valuing corporate securities: some effects of bond indenture provisions. J. Finance 31, 351-367.

Bossy, M., Gobet, E. And Talay, D. (2004). A symmetrized Euler scheme for an efficient approximation of reflected diffusions. J. Appl. Prob. 41, 877-889.

Davydov, D. And Linetsky, V. (2001). The valuation and hedging of barrier and lookback options under the CEV process. Manag. Sci. 47, 949-965.

Devroye, L. (1986). Nonuniform Random Variate Generation. Springer, New York.

Doob, J. L. (1949). Heuristic approach to the Kolmogorov-Smirnov theorems. Ann. Math. Statist. 20, $393-403$.

Fearnhead, P., Papaspiliopoulos, O. and Roberts, G. O. (2008). Particle filters for partially observed diffusions. To appear in J. R. Statist. Soc. B.

Gobet, E. (2000). Weak approximation of killed diffusion using Euler schemes. Stoch. Process. Appl. 87, 167-197.

Gobet, E. (2001). Euler schemes and half-space approximation for the simulation of diffusion in a domain. ESAIM Prob. Statist. 5, 261-297.

LÉPINGLE, D. (1995). Euler scheme for reflected stochastic differential equations. Math. Comput. Simul. 38, 119-126.

Lerche, H. R. (1986). Boundary Crossing of Brownian Motion. (Lecture Notes Statist. 40). Springer, Berlin.

LongstafF, F. A. AND Schwarz, E. S. (1995). A simple approach to valuing risky and floating rate debt. J. Finance 50, 789-819.

Merton, R. C. (1971). Theory of rational option pricing. Bell J. Econom. Manag. 4, 141-183.

ØKSEndal, B. K. (1998). Stochastic Differential Equations. An Introduction with Applications, 5th edn. Springer, Berlin.

Pötzelberger, K. And Wang, L. (2001). Boundary crossing probability for Brownian motion. J. Appl. Prob. 38, $152-164$.

Reiner, E. And Rubinstein, M. (1991). Breaking down the barriers. Risk 4, 28-35. 